\title{
Ergebnisse: Vor dem Studium
}

\subsection{Auswahl der Stipendiat*innen}

Ein Ziel des KAAD ist es, möglichst viele Studierende aus finanziell benachteiligten Familien zu fördern, um ihnen einen sozialen Aufstieg durch Bildung zu ermöglichen und damit zu einer Bekämpfung der Armut in den Herkunftsländern beizutragen. In unserer Onlineumfrage gab fast die Hälfte der Alumni an, dass sie aus einer Familie der unteren Mittelschicht stammen (49,7\%), ein weiteres Viertel aus der oberen Mittelschicht (24,9\%), ca. 12 Prozent ordneten ihre Familie der Unterschicht und 2 Prozent der Oberschicht in ihren Herkunftsländern zu. In Ghana stammen besonders viele Alumni aus finanzschwachen Familien, die traditionsgemäß ihr Einkommen in der Landwirtschaft erzielen. Über drei Viertel der interviewten ghanaischen Alumni und Stipendiat ${ }^{*}$ innen haben mindestens ein Elternteil, das in der Landwirtschaft arbeitet oder früher arbeitete. Häufig arbeiteten sogar beide Elternteile und auch die näheren Verwandten in der Landwirtschaft. Eine 27-jährige ghanaische Stipendiatin, die aus einem Dorf in der Nähe von Tamale im Norden Ghanas kommt, beschreibt ihre soziale Herkunft folgendermaßen:

„Ich komme aus einer Familie mit sehr geringem Einkommen. Meine Eltern sind Farmer außerhalb von Tamale. Als ich dort aufwuchs liefen die meisten Kinder noch nackt herum. Die meisten Familien konnten sich noch keine moderne Kleidung leisten. Heute ist das etwas besser geworden. Auch meine Eltern können sich gut von der eigenen Ernte ernähren und können zudem auch ein kleines Geschäft betreiben, um selbstgeerntetes Gemüse zu verkaufen, vor allem Mais. [...] Ich habe noch vier Geschwister, alle jünger, die wollen in der Landwirtschaft arbeiten. Für mich wäre das nichts, ich würde schon gerne in einer Stadt leben und arbeiten" (Interview mit einer ghanaischen Stipendiatin, Bonn, 2017).

S. Krannich und U. Hunger, Studierendenmigration und Entwicklung, https://doi.org/10.1007/978-3-658-32048-5_3 
Zudem haben viele ghanaische Alumni relativ viele Geschwister, teilweise bis zu zwölf. Dazu gehört auch ein Alumnus, der in armen Verhältnissen in einem Dorf im äußersten Nordwesten Ghanas aufgewachsen ist und sechs weitere Geschwister hat. Alle seine Vorfahren waren Subsistenzbauern mit einem geringen Einkommen. Er ist der erste Akademiker in der Familie und der erste, der im Ausland war. Mittlerweile ist er Professor an der KNUST-Universität in Kumasi und baut ein eigenes Haus für seine Familie mit zwei Kindern. Ein anderer Alumnus betont, dass das ländliche Leben in Ghana den Zugang zu Bildung erschwert:

„Die nächste Schule war über $140 \mathrm{~km}$ von meinem Heimatdorf entfernt, d.h. ich musste sehr früh meine Eltern verlassen, um in die Schule gehen zu können. Viele Kinder gehen deswegen nicht in die Schule, weil sie zu weit weg ist und sie nicht getrennt von ihren Eltern leben wollen und ein Umzug auch zu teuer ist. Das ist nicht gut, weil man als Kind seine Eltern braucht. So geht es vielen Kindern in Ghana. Ich habe gemerkt, dass man das ändern muss"(Interview mit einem ghanaischen Alumnus, Kumasi, Ghana, 2018).

Die interviewten Alumni aus Kolumbien stammen ebenfalls aus eher einkommensschwachen Familien. Ohne staatliche Unterstützung hätte man kaum eine Chance gehabt, ein Studium aufzunehmen. Ein kolumbianischer Alumnus sagte dazu:

„Nein. Ich stamme aus einer Familie, die wenige Möglichkeiten hatte. Meine Mutter war alleine. Sie hatte kein Geld mein Studium zu finanzieren. Meine Familie besteht aus vier Mitgliedern: meine Mutter und drei Kinder. Ich bin der jüngste davon. Die anderen zwei haben die Universität auch besucht, wobei sie ihr Studium am Abend gemacht haben, das heißt sie mussten am Tag arbeiten, um ihr Studium am Abend zu finanzieren. Ich war der einzige, der tagsüber studiert hat, und naturgemäß habe ich diesen ICETEX-Kredit ${ }^{9}$ dafür bekommen, aber ich habe nicht gearbeitet" (Interview mit einem kolumbianischen KAAD-Alumnus, Bogotá, Kolumbien, 2017).

Demgegenüber kommen die geförderten indonesischen Alumni aus eher wohlhabenderen Familien. Das hängt auch damit zusammen, dass viele von ihnen von katholischen Chinesen abstammen, die im 19. und 20. Jahrhundert auswanderten und erfolgreiche Unternehmen in Indonesien gründeten oder später relativ gut

9 ICETEX (Instituto Colombiano de Crédito Educativo y Estudios Técnicos en el Exterior Mariano Ospina Pérez) ist ein Studienkredit in Kolumbien, der dem deutschen BAFöG vergleichbar ist. 
bezahlte Stellen in der Energie- und Technologiebranche bekommen haben. Auch die nachfolgenden Generationen entschieden sich später für ein Studium eines MINT-Faches. Eine interviewte Alumna kommt zum Beispiel aus einer wohlhabenden Mittelschichtsfamilie. Beide Elternteile haben BWL in Jakarta studiert. Heute arbeitet der Vater im indonesischen Finanzministerium und die Mutter für eine indonesische Großbank. Sie konnten es sich daher leisten, ihre Tochter auf eine katholische Privatschule zu schicken (Interview mit einer indonesischen Alumna, Jakarta, Indonesien, 2017). Der Vater eines anderen Alumnus, der als Kind mit seinen Eltern aus China kam, hat Rechtswissenschaft in der javanesischen Stadt Bandung studiert und konnte es sich leisten, das Bachelorstudium seines Sohnes in Jakarta und auch einen Teil seines Masterstudiums in Deutschland zu finanzieren (Interview mit einem indonesischen Alumnus, Pontianak, Indonesien, 2017). Eine andere Alumna, die aus einer Juristenfamilie kommt, sagte:

\begin{abstract}
"I grew up in a village close to Yogyakarta. My mother worked as a lawyer, my grandpa too, and my father as an English teacher. My father died five years ago, and my mother is retired now and lives alone in a small house in Yogyakarta. But she always supported me and is still supporting me" (Interview mit einer indonesischen Alumna, Jakarta, Indonesien, 2017).
\end{abstract}

Die georgischen und palästinensischen Alumni kommen aus unterschiedlichen sozialen Verhältnissen. So sehen ungefähr die Hälfte der interviewten georgischen Stipendiat ${ }^{*}$ innen und Alumni ihre soziale Herkunft in der gebildeten urbanen Mittelschicht. In Palästina sind es sogar fast zwei Drittel der Interviewten. Eine Alumna aus Ramallah erklärte:

„Auch heute ist es noch so, dass man in Palästina seine Kinder nur in eine gute Schule und dann vielleicht sogar an eine Universität schickt, wenn man den Wert von Bildung begreift. Viele Eltern, die auf dem Land leben und die wirtschaftlichen Beziehungen in den Städten nicht verstehen, wollen ihre Kinder lieber in Handwerksberufen sehen. Das ist auch ok so, aber in den Städten, wo die gut ausgebildeten Fachleute leben, setzt man andere Prioritäten. Dort versucht man, dass die Kinder eine gute Ausbildung bekommen und auch studieren" (Interview mit einer palästinensischen Alumna, Ramallah, Palästina, 2016).

In allen untersuchten Ländern haben die KAAD-Alumni ähnliche religiöse Hintergründe. Die meisten Alumni kommen aus katholischen Familien: über 80 Prozent der interviewten Alumni und rund 70 Prozent der online befragten Alumni. Das liegt hauptsächlich an der katholischen Ausrichtung des KAAD, der in erster 
Linie die akademische Ausbildung in Deutschland und Karriere katholischer Studierender und Wissenschaftler*innen in den Herkunftsländern unterstützen will. $\mathrm{Zu}$ einem geringeren Teil (bis zu $30 \%$ pro Jahrgang) werden aber auch gezielt Studierende anderer Konfessionen gefördert, damit nicht ausschließlich die Konfessionszugehörigkeit über die Aufnahme in die Förderung entscheidet. So waren in unserer Online-Studie über 14 Prozent der Befragten Angehörige einer orthodoxen Kirche, die meisten davon der orthodoxen Kirche Georgiens. Weitere vier Prozent waren evangelisch, zwei sunnitisch, 0,5 Prozent schiitisch. Weitere sechs Prozent gehörten anderen Konfessionen an, wie buddhistischen und hinduistischen sowie kleinerer Konfessionen, wie äthiopisch-orthodox, maronitisch oder drusisch (KAAD-Jahresbericht 2018: 136ff.).

\subsection{Studium und Beruf im Herkunftsland}

Ein Aspekt der Förderpolitik des KAAD ist es zudem, dass vorwiegend internationale Studierende gefördert werden, die mindestens bereits einen Bachelor-Abschluss in ihrem Herkunftsland erworben haben, weil dadurch die Studierenden bereits ein gewisses Alter erreicht haben und universitäre Erfahrungen mitbringen und sich somit wahrscheinlich auch leichter mit einem Leben und Studium im Ausland zurecht finden (Interview mit einem Vertreter des KAAD, Bonn, 2016; KAAD 2010ff.). Zudem geht man davon aus, dass sich dadurch auch die Bereitschaft unter den Stipendiat*innen erhöht, nach dem Studium in Deutschland wieder in das Herkunftsland zurückzukehren, vor allem weil man älter ist und mehr Beziehungen zum Herkunftsland aufgebaut hat (Interview mit einem Vertreter des KAAD, Bonn, 2016). Rund 80 Prozent der befragten Alumni hatte dementsprechend entweder ein Bachelor- oder ein Masterstudium im Herkunftsland absolviert. Weitere 20 Prozent hatten sogar einen Doktortitel im Herkunftsland erworben und sind danach als Postdoktorand ${ }^{*}$ innen bzw. Gastwissenschaftler*innen an eine deutsche Universität oder Hochschule gegangen. Darunter waren auch Stipendiat*innen, die bereits als Professor*innen im Herkunftsland gearbeitet haben. Drei Viertel der befragten KAAD-Stipendiat*innen und Alumni haben ihre Hochschulzugangsberechtigung im Herkunftsland erworben. Das andere Viertel hat entweder eine Hochschulzugangsberechtigung an einem Studienkolleg in Deutschland erworben (knapp über $20 \%$ ) oder in einem anderen EU-Land (über zwei Prozent) bzw. in einem anderen Land außerhalb der EU (knapp drei Prozent).

Die Studiengänge und Fachrichtungen der KAAD-Alumni in den Herkunftsländern vor dem Aufenthalt in Deutschland sind dabei sehr vielfältig. Insgesamt 
wurden 59 verschiedene Studiengänge von den Befragten in unserer Onlineumfrage angegeben. Gut 38 Prozent der Befragten studierten/forschten im Bereich Sozialwissenschaften. Davon die meisten in den Fächern Politikwissenschaft, Volkswirtschaftslehre und Kommunikationswissenschaft. Weitere knapp 38 Prozent waren in einem MINT-Studiengang eingeschrieben, vor allem in den Fächern Biologie, Ingenieurwissenschaft und Architektur. Weitere knapp 20 Prozent studierten oder forschten in einem Fach der Geisteswissenschaften, vor allem Geschichte, Deutsche und Englische Philologie, darunter auch sog. „Orchideenfächer“ wie Orientalistik und Arabistik. Knapp fünf Prozent waren schließlich für ein Studium im Bereich Kunst und Musik in Deutschland. Diese Förderzahlen spiegeln auch die akademische Philosophie des KAAD wider, der nicht nur internationale Studierende in „ökonomisch nützlichen " Fächern fördern möchte, sondern möglichst in der gesamten akademischen Bandbreite (Interviews mit Vertretern des KAAD, Bonn, 2016).

Die meisten Studierenden haben sich zudem bereits vor ihrer Förderung in Deutschland mit entwicklungsrelevanten Themen in ihren Abschlussarbeiten im Herkunftsland auseinandergesetzt, die sie häufig in ihrem späteren Studium in Deutschland weiterentwickelten. Die meisten ghanaischen Alumni haben sich bereits in ihrem Studium in Ghana mit Umwelt-, Energie- und Landwirtschaftsfragen auseinandergesetzt. Zum Beispiel verfasste ein ghanaischer Alumnus, der von 2007 bis $2010 \mathrm{im}$ Studiengang „Geography and Regional Development“ an der Accra University Legon studierte, eine Bachelorarbeit zum Thema „Müllmanagement und -entsorgung in der ghanaischen Region Tema“. Dabei ging er der Frage nach, wie Haushalte mit einem geringen Einkommen es schaffen können, ihren Müll auf kostengünstige und nachhaltige Weise zu entsorgen. Hintergrund ist, dass die meisten Kommunen in Ghana nicht genug Geld haben, um eine kommunale Müllabfuhr zu organisieren (Interview mit einem ghanaischen Alumnus, Accra, Ghana, 2018).

Ein anderer ghanaischer Alumnus beschäftigte sich schon in den 1990er Jahren in seiner Bachelorarbeit im Fach Ökologie an der Kwame Nkrumah University of Science and Technology (KNUST) in Kumasi mit den Wasserkreisläufen im ghanaischen Regenwald und welchen Umwelteinflüssen sie unterliegen. Bereits damals war das Thema Wasserknappheit und die Gewinnung und Weiterleitung von Wasser ein vieldiskutiertes Thema in Ghana. Auch während seines Masterund Promotionsstudiums später an der Universität Bremen setzte er sich mit dem Thema Wasser auseinander. Nach seiner Rückkehr nach Ghana wurde er Dozent im Fach Ökologie an der KNUST in Kumasi, wo er früher sein Bachelorstudium absolvierte. Hier beschäftigt er sich weiterhin mit Fragen des Umweltschutzes und der Wasserqualität in ghanaischen Flüssen und Seen (Interview mit einem ghanaischen Alumnus, Kumasi, Ghana, 2018), worauf wir weiter unten noch eingehen werden. 
Auch in den anderen Ländern unserer Fallstudie lassen sich Beispiele finden, bei denen sich Alumni bereits in ihren Bachelorstudiengängen mit Themen auseinandersetzen, die für ihr Land entwicklungsrelevant sind oder sein können. Ein kolumbianischer Alumnus studierte z. B. vor seinem Studium in Deutschland Rechtswissenschaft an der Universidad del Libre in Bogotá mit den Schwerpunkten Strafrecht und Strafprozessrecht in Kolumbien, insbesondere mit Bezug auf die Strafverfolgung im Rahmen des bewaffneten Konflikts in Kolumbien. Diese Studien vertiefte er zunächst im Rahmen eines Aufbaustudiums an der Universidad Externado in Bogotá und anschließend in einem zweijährigen Master of Laws-Studium (LL.M.) an der Albert-Ludwigs-Universität in Freiburg. In seiner Masterarbeit verglich er das kolumbianische mit dem deutschen Strafrecht, in dem er Vorbildcharakter für das kolumbianische Strafrecht sah und wichtige Aspekte für die Konzipierung der Strafgesetze in Kolumbien vorschlug.

Ein anderer kolumbianischer Alumnus studierte ebenfalls Jura in Kolumbien, bevor er zum Studium nach Deutschland ging. In seiner Abschlussarbeit an der privaten Universidad Externado Colombia in Bogotá, die er mit dem rückzahlungspflichtigen Stipendium des kolumbianischen Staats, dem o. g. ICETEX-Stipendium, finanzierte, beschäftigte er sich mit dem Thema „Underwriting in Kolumbien“. Dabei definierte er "Underwriting" als einen Vertrag im Börsengeschäft, nach dem z. B. auch Börsenmakler Emissionen einer Unternehmensaktie in eigenem Namen unternehmen dürfen. Da dies in den 1990er Jahren in Kolumbien ein noch neues Thema war, hat er nach seinem Studium auch schnell eine Anstellung bei der Bankenaufsichtsbehörde in Bogotá bekommen (Interview mit einem kolumbianischen Alumnus, Bogotá, Kolumbien, 2017).

Verschiedene Alumni haben nicht nur studiert, sondern auch schon für einen gewissen Zeitraum Berufserfahrungen gesammelt, bevor sie nach Deutschland gekommen sind. So hat zum Beispiel ein ghanaischer Stipendiat nach seinem Masterabschluss bereits für ein paar Jahre als Lecturer im Bereich Landwirtschaft und Land-Management an der Wa University gearbeitet, bevor er nach Deutschland ging, um dort zu promovieren (Interview mit einem ghanaischen Stipendiaten, Skype, 2016). Eine Alumna aus Indonesien arbeitete bereits von 2005 bis 2007 als Juradozentin an der privaten katholischen KAAD-Partneruniversität Atma Jaya in Jakarta. Danach nahm sie ein Promotionsstudium an der Ludwig-MaximiliansUniversität in München zum Thema „Wettbewerbsrecht in Indonesien und Deutschland im Vergleich" auf. In ihrer Lehre bot sie Seminare zum Wirtschaftsrecht in Indonesien und im internationalen Vergleich an (Interview mit einer indonesischen Alumna, Jakarta, Indonesien, 2017). Eine andere kolumbianische Alumna lehrte und forschte bereits über zehn Jahre an verschiedenen Universitäten in Bogotá, bevor sie ihr Promotionsstudium in Deutschland begann. 
Einige Stipendiat ${ }^{*}$ innen und Alumni sammelten auch bereits Berufserfahrungen in entwicklungsrelevanten Bereichen. Ein ghanaischer Stipendiat arbeitete z.B. von 2003 bis $2009 \mathrm{im}$ „Ghana Descent Work Program“, das von der International Labor Organization (ILO) finanziert wurde. Dabei war er für die Beobachtung der Entwicklung des lokalen Regierungshandelns zuständig, das durch das Programm der ILO effektiver und demokratischer gestaltet werden sollte. Er hat dieses Programm in einigen Kommunen evaluiert - wie z. B. in zwei Bezirken in der Nähe von Accra - und sollte feststellen, inwieweit es den lokalen Behörden gelungen ist, auch lokale Unternehmen und zivilgesellschaftliche Akteure in ihre Politik einzubeziehen. Am Ende waren die Ergebnisse aber sehr unbefriedigend für ihn, weil die ILO-Beauftragten, obwohl sie auch aus Ghana stammten, allerdings aus der Großstadt, die lokale Politikkultur in dieser ländlichen Region Ghanas seiner Meinung nach nicht verstanden. Dort gebe es klare Hierarchien und geerbte Autoritäten, die man nicht einfach demokratisch reformieren könne, indem man Unternehmen und NGOs in die Entscheidungsprozesse miteinbeziehe. Das Programm wurde dementsprechend nicht von den lokalen Eliten und großen Teilen der Gemeindebevölkerung angenommen. Danach arbeitete er noch für eine kurze Zeit für das "Ghana Poverty Reducing Program“ (Interview mit einem ghanaischen Stipendiaten, Skype, 2016).

Ein anderer ghanaischer Stipendiat arbeitete vier Jahre für die Consulting-Firma Ernest and Young in Ghana, bevor er zum Studium nach Deutschland ging. Dabei hat er vor allem die ghanaische Regierung und staatliche Institutionen beraten, insbesondere in Bezug auf Entwicklungsprojekte in Ghana. Dabei konzentrierte er sich vor allem auf landwirtschaftliche Projekte, weil es seiner Meinung nach mehr technologische Entwicklung und den Einsatz guter Technologien in Ghana brauche (Interview mit einem ghanaischen Stipendiaten, Bonn, 2017).

Ein weiterer ghanaischer Alumnus hat neben seinem Bachelor- und Masterstudium an der KNUST in Kumasi als Farmhelfer in einem kleinen bäuerlichen Betrieb gearbeitet. Dabei hat er viel praktische Erfahrung für sein späteres Dissertationsthema gesammelt und viel gelernt über die Nöte der Farmer und deren Probleme mit ihren Agrarprodukten gegenüber europäischen Konkurrenten wettbewerbsfähig zu bleiben. Geholfen hat er vor allem beim Zwiebel- und Möhrenanbau. Während dieser Zeit fasste er den Entschluss, später selbst Farmer zu werden, anstatt eine wissenschaftliche Laufbahn einzuschlagen. Das Studium und die Promotion hat er nur gemacht, um mehr theoretisches Wissen über landwirtschaftliche Berufe zu sammeln. Er plant sich einmal als Farmer in der Eastern Region oder Ashanti Region in Ghana niederzulassen. Er sagte dazu: 
"I think, well-educated guys should become farmers, because they don't only know how to do farming, but also know how to deal with big companies and state institutions. They know how to communicate with them. And in my special case, I also know how to communicate with European people, because I studied there, and I know how they think. This could help to improve the situation of Ghanaian farm companies in Europe and how to sell goods there" (Interview mit einem ghanaischen Alumnus, Skype, 2017).

Auch eine andere KAAD-Alumna aus Ghana hat während ihres Studiums bereits Berufserfahrungen gesammelt. Während ihres Bachelorstudiums in Agriculture Technology an der Universität Tamale machte sie ein mehrwöchiges Praktikum beim ghanaischen Landwirtschaftsministerium. In diesem Rahmen besuchte sie mit zuständigen Ministern verschiedene Farmer und konnte sich auch selbst mit ihnen unterhalten und ein Bild von der aktuellen Lage machen. Auch später während ihres Masterstudiums in Deutschland beschäftigte sie sich mit der Situation von Kleinbauern in Ghana und anderen afrikanischen Ländern (Interview mit einer ghanaischen Alumna, Skype, 2017).

Ein anderer Alumnus aus Ghana wiederum sammelte berufliche Erfahrungen in der Telekommunikationsbranche. Nach dem Bachelorstudium im Fach Electrical Engineering an der KNUST in Kumasi arbeitete er von 2004 bis 2008 für die für die südafrikanische Telekommunikationsfirma MTN Group, wo er Ideen für sein späteres Studium in Deutschland sammelte (Interview mit einem ghanaischen Alumnus, Accra, Ghana, 2018).

Alumni aus Indonesien sammelten ebenfalls bereits Berufserfahrungen im Heimatland, bevor sie sich für ein Studium in Deutschland aufmachten. Ein interviewter Alumnus arbeitete nach seinem Bachelorstudium in den 1990er Jahren für zwei Jahre für die indonesische Tamara Bank. Er war dort vor allem für die Entwicklung von Marketingstrategien und die Ausgabe von VISA- and MasterKreditkarten zuständig. Dabei entwickelte er insbesondere Marketingstrategien für Kreditkarten, was Ende der 1990er in Indonesien ein enormer Wachstumsmarkt war (Interview mit einem indonesischen Alumnus, Jakarta, Indonesien, 2017). Eine andere indonesische Stipendiatin arbeitete vor ihrem Studium in Deutschland für das indonesische Gesundheitsministerium. Ihre Aufgabe war es, dabei zu helfen, eine allgemeine Gesundheitsversicherung für an HIV erkrankte Menschen zu konzipieren und zu implementieren. Das Problem bestand vor allem darin, dass viele Versicherungen sich weigerten, die Kosten für die HIV-Behandlung zu erstatten. So gab es viele Auseinandersetzungen zwischen Patient ${ }^{\star}$ innen und Versicherungsunternehmen, die häufig auch gerichtlich ausgetragen wurden. Eine allgemeine Gesundheitsversicherung für HIV-kranke Menschen wurde in Indonesien erst 
im Jahr 2014 eingeführt (Interview mit einer indonesischen Stipendiatin, Skype, 2017). Eine weitere indonesische Stipendiatin, die später Gesang an der Musikhochschule in Hannover studierte, absolvierte nach ihrem Musik-Bachelorstudium an der Hochschule für Musik, Theater und Medien Hannover eine künstlerische und pädagogische Ausbildung und arbeitete zudem für zwei Jahre als Lehrerin und Chorleiterin an einem Gymnasium in Jakarta (Interview mit einer indonesischen Stipendiatin, Skype, 2017).

In Kolumbien gibt es auch einige Alumni, die bereits Berufserfahrungen im Herkunftsland sammelten, bevor sie zum Studieren nach Deutschland gingen. Ein Jurastudent, der zuvor seine Abschlussarbeit zum „Underwriting-Vertrag“ in Bogotá geschrieben hatte, hat direkt im Anschluss an das Studium eine Anstellung bei der kolumbianischen Bankenaufsichtsbehörde bekommen. Dort arbeitete er von 1986 bis 1989. Danach ging er für ein Jurastudium nach Deutschland. Ein anderer kolumbianischer Alumnus unterrichtete nach seinem Bachelorabschluss Literatur an einer US-amerikanischen Privatschule in Bogotá. Zudem engagierte er sich für die NGO „Mimito de Dios“, die allgemeinbildenden Unterricht für hilfsbedürftige Kinder und Jugendliche aus finanzschwachen Familien anbietet. Dort unterrichtete er unter anderem Geschichte und Philosophie. Danach ging er im Jahr 2008 nach Deutschland, um in Frankfurt an der Goethe-Universität im Fach Religionsphilosophie zu promovieren (Interview mit einem kolumbianischen Alumnus, Bogotá, Kolumbien, 2017).

Auch ein anderer Stipendiat sammelte bereits vor seinem Studium in Deutschland Erfahrungen als Lehrer in Kolumbien. Er unterrichtete fast acht Jahre Englisch an einem Gymnasium in Bogotá. Bereits zuvor während seines Anglistikstudiums an der Universidad Nacional in Bogotá, wo er einen Bachelorabschluss erwarb, gab er Kindern und Jugendlichen, aber auch Erwachsenen Nachhilfeunterricht (Interview mit einem kolumbianischen Stipendiaten, Skype, 2017). Eine weitere Stipendiatin aus Kolumbien, die ein Lehramtsstudium für Spanisch und Deutsch an einer Universität in Medellin abschloss, arbeitete bereits acht Jahre am Lehrerfortbildungszentrum MOVA der Stadt Medellin, bevor sie zum Studium nach Deutschland ging (Interview mit einer kolumbianischen Stipendiatin, Heidelberg, 2018). Eine weitere Alumna aus Bogotá arbeitete vor ihrem Studium an der Universität Duisburg für ca. ein Jahr im Rahmen eines Projekts der Internationalen Organisation für Migration (IOM) in Kolumbien. Im Rahmen des Projekts arbeitete sie mit verschiedenen ethnischen Gruppen sowie hilfsbedürftigen Frauen in ländlichen Gegenden zusammen, die sehr stark vom kolumbianischen Bürgerkrieg betroffen waren (Interview mit einer kolumbianischen Alumna, Bogotá, 2017).

Die georgischen Stipendiat*innen und Alumni waren vor ihrem Studium oder Forschungsaufenthalt in Deutschland vor allem im juristischen oder aka- 
demischen Bereich tätig. Ein Stipendiat arbeitete vor seinem Promotionsstudium an der Universität Hannover für das georgische Justizministerium. Im Rahmen dieser Tätigkeit beschäftigte er sich bereits mit deutschen Gesetzen, ehe er von 2013 bis 2015 - vom KAAD gefördert - zum Thema „Deutsches und georgisches Jugendstrafrecht" promovierte. Bereits im Rahmen seiner Arbeit für das Justizministerium überprüfte er, inwieweit deutsche Grundsätze des Jugendstrafrechts in das georgische Rechtssystem implementiert werden könnten. Damals wurde er nur aufgrund seiner guten Deutschkenntnisse eingestellt, weil man händeringend einen Übersetzer mit Rechtskenntnissen suchte. Zuvor erwarb er das juristische Staatsexamen an der Staatlichen Universität Tiflis (Interview mit einem georgischen Stipendiaten, Skype, 2017).

Eine Alumna, die erst kürzlich von ihrem Masterstudium „Deutsches und Europäisches Recht“ (LL.M.) an der Humboldt-Universität zu Berlin nach Georgien zurückkehrte, arbeitete vorher als Rechtsberaterin in der Nationalagentur für das staatliche Eigentum (AdöR) in Tiflis (Interview mit einer georgischen Alumna, Berlin, 2016). Ein anderer Alumnus, der inzwischen Juraprofessor an der East European University in Tiflis ist und für einen dreimonatigen Forschungsaufenthalt am Max-Planck-Institut für Ausländisches und Öffentliches Recht in Heidelberg vom KAAD gefördert wurde, arbeitete früher als Rechtsberater am Obersten Gerichtshof (Interview mit einer georgischen Alumna, Tiflis, 2016).

Ein weiterer Alumnus arbeitete vor seinem Promotionsaufenthalt an der Humboldt-Universität in Berlin von 2013 bis 2015 als Rechtsberater in der Abteilung für Internationale Beziehungen des georgischen Parlaments sowie im Ausschuss für Verteidigung und Sicherheit. Zudem arbeitete er zur gleichen Zeit neben seinen Forschungsprojekten in der Vertrauensgruppe zu Korruptionsfragen des Nationalen Parlaments in Tiflis mit. Dabei beschäftigt er sich mit der Aufklärung verschiedener Korruptionsfälle. Es kam ihm darauf an, demokratische Rechtsprinzipien und Gesetze auch im Politikalltag umzusetzen. Seit seiner Rückkehr im Jahr 2015 arbeitet er wieder als Staatsanwalt in der Abteilung für Überwachung der staatsanwaltlichen Arbeit und strategischen Entwicklung der Generalstaatsanwaltschaft Georgiens (Interview mit einem georgischen Alumnus, Tiflis, 2016).

Ein weiterer Alumnus arbeitete bereits als 23-Jähriger während seines Studiums an der Staatlichen Universität Tiflis als Lektor für Internationales sowie georgisches Verfassungsrecht am Staatlichen Institut für ökonomische Beziehungen in Tiflis (TEUSI). Während seiner Promotion war er von 2009 bis 2013 als Mitglied im parlamentarischen Ausschuss für Kulturpflege tätig. In diesem Rahmen arbeitete er maßgeblich an einem Gesetzesentwurf zum Schutz des nationalen immateriellen Kulturerbes in Georgien mit. Dabei musste er insbesondere die Recherche zu georgischen Kulturgütern besorgen sowie einen Vergleich mit Gesetzen in anderen 
Ländern zu diesem Thema durchführen. Auch die Implementierung der UNESCOWeltkulturerbe-Richtlinien in das Gesetz gehörte zu seinen Aufgaben. Von November 2015 bis April 2016 wurde er für einen Forschungsaufenthalt im Rahmen seines Dissertationsprojekts im Bereich Rechtsphilosophie an der Universität Bonn vom KAAD gefördert (Interview mit einem georgischen Alumnus, Skype, 2017).

Die meisten palästinensischen KAAD-Alumni, die wir interviewt haben, waren relativ jung als sie nach Deutschland zum Studieren kamen und hatten daher kaum berufliche oder akademische Vorerfahrungen. Viele von ihnen hatten zuvor einen Bachelor-Abschluss an einer Universität in der Westbank (v. a. in Bethlehem und Ramallah) gemacht. Ein paar wenige aber auch in Israel, wie z. B. eine Alumna, die direkt vor ihrem Studium in Heidelberg einen Bachelor an der renommierten Hebräischen Universität in Jerusalem erwarb (Interview mit einer palästinensischen Alumna, Tel Aviv-Jaffa, Israel, 2016). Die einzige Alumna, die bereits vor ihrem KAAD-geförderten Medizinstudium im Ausland studierte, war eine 68-jährige Ärztin, die in den 1970er Jahren ein Medizinstudium in Rumänien begann, es aber aus privaten Gründen bereits nach zwei Semestern abbrach (Interview mit einer palästinensischen Alumna, Ramallah, Palästina, 2016).

Einige Alumni haben vor ihrem Studium in Deutschland auch außerhalb ihres Heimatlandes studiert oder Berufserfahrungen gesammelt. So hat z. B. eine ghanaische Alumna vor ihrem Promotionsstudium in Bonn einen Masterabschluss im Fach „Landwirtschaft“ an der Universität in Den Haag im Jahr 2007 erworben. Dort hat sie die ersten Kontakte für ihr späteres Promotionsstudium an der Universität Bonn geknüpft (Interview mit einer ghanaischen Alumna, Accra, Ghana, 2018). Ein kolumbianischer Alumnus hat direkt nach seinem Bachelorabschluss an der privaten katholischen KAAD-Partneruniversität Javeriana in Bogotá einen Masterstudiengang in „Religious Studies“ an der Lancaster University in England von 2002 bis 2004 angehängt. In seiner Masterarbeit beschäftigte er sich mit dem interreligiösen Dialog zwischen indigenen Gruppen und mestizischen Christen in Lateinamerika. Dabei analysierte er die Bedingungen für den Dialog zwischen indigenen Weltanschauungen und Christentum in Lateinamerika aus philosophischer Sicht. Danach ging er erst einmal für ein paar Jahre zurück nach Kolumbien, bevor er 2008 nach Deutschland kam, um an der Goethe-Universität Frankfurt im Fach Religionsphilosophie zu promovieren (Interview mit einem kolumbianischen Alumnus, Bogotá, Kolumbien, 2017). 


\subsection{Gründe für ein Studium in Deutschland}

Es gibt viele Gründe, warum sich internationale Studierende für ein Studium in Deutschland entscheiden. Die drei Hauptgründe in unserer Studie waren: bessere Studienangebote und Forschungsbedingungen in Deutschland (1), bereits bestehende Kontakte nach Deutschland (2) und ein persönliches Interesse an Deutschland (3). In einigen Fällen spielten auch mehrere Gründe gleichzeitig eine Rolle (4).

\subsubsection{Studienangebote und Forschungsbedingungen in Deutschland}

Der Hochschulstandort Deutschland ist für die befragten Alumni attraktiv. Das liegt vor allem an den im Vergleich zu den Heimatländern größeren Studienangebot in Deutschland. Ein Alumnus aus Kolumbien beschrieb diesen Gegensatz in Bezug auf die Situation in Kolumbien in den 1980er Jahren folgendermaßen:

„Kolumbien war damals noch nicht so weit wie heute. Auch heute ist Kolumbien natürlich noch weit hinter Deutschland und den meisten anderen westlichen Ländern, aber damals war der Abstand noch größer. Es gab kaum Förderung für Forschungsprojekte. Man hat nicht erkannt, dass Wissenschaft wichtig ist, um das Land zu entwickeln. Heute hat man das zumindest schon mal erkannt, aber es fehlen noch die Gelder. Ich glaube, dass das durch den internationalen Austausch immer besser wird, und auch durch die steigende Vernetzung der kolumbianischen Universitäten mit europäischen Universitäten. [...] Auf der anderen Seite hatte ich Kolumbien nicht als Entwicklungsland gesehen. Ich kannte Ecuador und Venezuela, und im Vergleich zu diesen beiden Nachbarländern war Kolumbien auf jeden Fall besser, viel weiter, und viel mehr Universitäten. In Deutschland ist mir dann bewusst geworden, dass wir doch noch nicht so weit sind, da fehlt noch einiges" (Interview mit einem kolumbianischen Alumnus, Bogotá, Kolumbien, 2017).

Daher wählten viele Alumni bewusst auch Deutschland als Studien- und Forschungsland aus, um sich akademisch weiterzubilden und um auf ihrem Gebiet tiefer einsteigen zu können. Eine andere kolumbianische Alumna sagte dazu:

„Unsere Wissenschaft ist noch nicht so weit. Da brauchen wir noch viele Jahre. Das war ja auch der Grund, warum mein Mann und ich in einem anderen Land promovieren wollten. In einem Land wie Deutschland, in dem es die 
wissenschaftlichen Strukturen gibt, um sich zu spezialisieren und in die Tiefe forschen zu können. Kolumbien ist in dieser Hinsicht definitiv noch ein Entwicklungsland. Deswegen wollen auch so viele im Ausland studieren. Wissen erwerben, das es in Kolumbien noch nicht gibt" (Interview mit einer kolumbianischen Alumna, Medellin, Kolumbien, 2017).

Das Kriterium guter Forschungs- und guter Studienbedingungen an deutschen Universitäten wurde auch von vielen anderen Alumni in den Interviews genannt, warum sie sich für ein Studium oder Forschungsaufenthalt in Deutschland entschieden haben. Ein ghanaischer Alumnus hat sich zum Beispiel speziell für ein Studium an der Universität Bonn entschieden, weil dort der Studiengang „Geography of Environmental Risks and Human Security “ in Kooperation mit der Universität der UNO angeboten wird. Über diesen Studiengang hat er von einem Professor in Accra gehört. Dieser Studiengang kombinierte alle Studienschwerpunkte des interviewten Alumnus und vermittelt vor allem eine globale Perspektive auf die Themen Umweltschutz und Nachhaltigkeit:
"I always wanted to have a global approach to the issues climate change, hu- man security, and risk management, because we can deal with them only on a global level, and not from a national or local level. That's why I decided to study in Bonn, taking classes at the University of Bonn and at the University of the United Nations. There is also an UN university campus in Accra. I want to cooperate with them when I'm back in Accra" (Interview mit einem ghanaischen Alumnus, Bonn, 2018).

Eine andere ghanaische Stipendiatin wollte Landwirtschaft insbesondere in Deutschland studieren, weil es in Deutschland die modernste Landwirtschaftstechnologie gebe und eine ausgeprägte biologische und nachhaltige Landwirtschaft betrieben werde. Das Wissen dazu wollte sie in Deutschland erwerben und mit nach Ghana bringen. Sie sagte dazu:

„Deutschland ist ein führendes Land in der landwirtschaftlichen Produktion. Die deutschen Bauern haben die modernste Technologie, um ihre Äcker zu bewirtschaften und Nutztiere zu züchten. Und dabei achten sie auch noch auf die Umwelt. Viele deutsche Bauern sind organic farmers. Das finde ich sehr gut, weil es eben gesund und nachhaltig ist. Ich glaube die Deutschen sind führend weltweit darin. Davon kann ich viel lernen. Und nachhaltige Bio-Landwirtschaft kann eben auch ein Modell für Ghana sein. [...] Dieses Bewusstsein 
und Wissen über nachhaltige biologische Landwirtschaft möchte ich mit nach Ghana bringen"(Interview mit einer ghanaischen Stipendiatin, Skype, 2017).

Eine Stipendiatin aus Indonesien hat sich für ein Studium der Gesundheitswissenschaft in Deutschland entschieden, weil es in Deutschland eine lange Tradition eines staatlich gesteuerten Gesundheitswesens gibt, das zu den besten der Welt gehöre (Interview mit einer indonesischen Stipendiatin, Skype, 2017). Ähnlich war es auch mit einem Alumnus aus Kolumbien, der sich für ein Jurastudium mit dem Schwerpunkt Strafrecht an der Universität Freiburg entschied, weil das deutsche Strafrecht Vorbildcharakter für Kolumbien hat. Er erklärte:

„Deutschland ist sehr wichtig für das kolumbianische Strafrecht. Das hatte einen großen Einfluss auf Kolumbien. [...] In den 1980er Jahren gab es eine Gruppe von kolumbianischen Professoren, die in Deutschland studiert haben und sie waren Berater im Parlament bei der letzten Überarbeitung oder Ausarbeitung des kolumbianischen Strafgesetzbuches. Dieses Gesetzbuch hat seinen dogmatischen Einfluss vom deutschen Recht. Es ist sehr ähnlich mit dem deutschen Strafgesetzbuch. Und deswegen waren auch viele deutsche Juraprofessoren in den letzten 20 Jahren in Kolumbien aktiv. So hat diese Verbindung angefangen. Jedes juristische Fachgebiet hat einen besonderen Einfluss von bestimmten Ländern. Zum Beispiel für Verwaltungsrecht ist es die französische Schule. Komischerweise für Strafprozessrecht haben wir einen US-amerikanischen Einfluss. Aber für Strafrecht ist es Deutschland [...]. Und viele bekannte deutsche Professoren waren hier in Kolumbien, insbesondere Klaus Roxin. Er ist sehr bekannt, er ist einer der wichtigsten Strafrechtler der Welt. [...] Ich habe angefangen die deutsche Sprache an der Uni hier in Kolumbien zu lernen, danach am Goethe-Institut. Und hier in Kolloquien habe ich einige deutsche Professoren kennengelernt. Ich habe ihnen E-mails geschickt und erklärt, warum ich in Deutschland studieren möchte. Und ich hatte drei Möglichkeiten, und zwar Konstanz, Freiburg und München. Warum habe ich mich für Freiburg entschlossen? Ein wichtiger Grund war, in Freiburg ist das Max-Planck-Institut für Internationales Strafrecht, da ist eine der größten Bibliotheken für Strafrecht der Welt, und das LL.M.-Programm dauert dort zwei Jahre, normalerweise ist LL.M. nur für ein Jahr. Das waren gute Gründe für mich nach Freiburg zu gehen. Und nicht nur Strafrecht, sondern auch öffentliches Recht als Fach zu haben. Auch die Region im Schwarzwald finde ich sehr schön, meine Verwandten wohnen in der Schweiz, also auch sehr nah. München war auch interessant, aber ein bisschen zu teuer, der Stipendienbetrag hätte dafür nicht ausgereicht, für eine Wohnung in München. Ich glaube, das 
war eine richtige Entscheidung. Ich habe immer noch Verbindungen mit der Uni Freiburg, mit dem Max-Planck-Institut. Und ich habe zwei Forschungsaufenthalte nach meinem LL.M.-Studium auch dort gemacht. Letztes Jahr an der Universidad del Libre, wo ich auch Privatdozent bin, haben wir einen großen internationalen Kongress organisiert zu Ehren von Professor Klaus Roxin. Wir haben viele deutsche Professoren eingeladen, aus München, Freiburg und so weiter. Die wurden alle eingeladen aufgrund meiner Kontakte, die während meines Studiums in Deutschland und meiner Forschungen entstanden sind. Ich kann sagen, die Türen sind weit offen mit Freiburg"(Interview mit einem kolumbianischen Alumnus, Bogotá, Kolumbien, 2017).

Ein anderer Stipendiat aus Ghana hat sich bewusst für ein Studium in Deutschland entschieden, weil er während der Teilnahme an einer Summer School vor ein paar Jahren in Dortmund erfahren hat, dass es viele internationale Entwicklungsorganisationen in Deutschland gibt, die sich für die Entwicklung in Afrika engagieren. Auch in Ghana hätten die deutschen Entwicklungsorganisationen, wie die GIZ, eine gute Reputation. Er versprach sich von einem Studium in Deutschland, dass er mit vielen Organisationen in Kontakt kommt und möglicherweise nach dem Studium für eine deutsche Organisation in Ghana arbeiten könnte (Interview mit einem ghanaischen Stipendiaten, Skype, 2017).

Auch eine indonesische Stipendiatin hat sich für ein Studium in Deutschland entschieden, weil deutsche Architektur Weltruf genieße und ein Bekannter von ihr selbst Architektur in Deutschland studiert hat. Sie tat das, obwohl ihre Schwester ein Studium in Australien empfohlen hat, weil sie dort sehr positive Erfahrungen gemacht hatte (Interview mit einer indonesischen Stipendiatin, Skype, 2017). Auch viele palästinensische KAAD-Alumni haben sich bewusst für ein Medizinstudium in Deutschland entschieden, weil die medizinische Ausbildung in Deutschland einen sehr guten Ruf in Palästina genießt. Ein palästinensischer Alumnus hat es folgendermaßen auf den Punkt gebracht:

„Deutschland hat eine lange Tradition in der Medizin. Viele medizinische Erfindungen und Entdeckungen wurden in Deutschland gemacht, vor allem in Berlin. Fast jeder Palästinenser kennt das Krankenhaus Charité, vor allem von den alten Beziehungen in der DDR. Aber auch schon davor, als viele MedizinNobelpreisträger aus Deutschland kamen. Davon profitiert Deutschland noch heute, und mir war klar, dass ich in Deutschland auch eine gute Medizinausbildung erhalten würde. Das war eine gute Entscheidung in Deutschland zu studieren" (Interview mit einem palästinensischen Alumnus, Bethlehem, Palästina, 2016). 
Neben den relativ gering entwickelten Studien- und Forschungsbedingungen gaben einige Alumni auch ganz konkret die schlechten und widrigen politischen Bedingungen im Heimatland an. Dies war zum Beispiel in Indonesien Ende der 1990er Jahre der Fall, als viele Studierende, die es sich finanziell leisten konnten, oder eben ein Stipendium bekamen, ins Ausland gingen. So erklärte zum Beispiel ein Alumnus des KAAD, dass der Zusammenbruch der Diktatur Suhartos und die damit einhergehende politische Instabilität und wirtschaftliche Unsicherheit, ihn dazu bewog, Indonesien zu verlassen und ein Studium im Ausland zu absolvieren:

\begin{abstract}
"I wanted to leave at that time, because the political and economic situation was very difficult. The country was in a revolution, and many jobs were not safe. Many lost their jobs or had to be afraid to lose them. There were also riots on the streets of Jakarta. Very insecure times. In these troublesome times, I decided to leave the country and get more education, and then come back when the economic and political troubles are over. [...] Only after a few years, we could see that the change was good. Already in 2000, the economy was growing as fast as never before. New investments from abroad came to Indonesia, because it was not a totalistic regime anymore, and foreign companies felt safer in Indonesia, and the markets opened to foreign companies. [...] Also, the people felt safer in Indonesia, because their rights were more protected in a democratic state of Indonesia. Before 1998, they were afraid to say anything. After 1998, they had more freedom, and freedom of speech" (Interview mit einem kolumbianischen Alumnus, Jakarta, Indonesien, 2017).
\end{abstract}

Letztendlich entschied er sich für ein Studium der Ingenieurwissenschaft in Deutschland, das in diesem Gebiet zudem weltweit eine gute Reputation genießt.

\title{
3.3.2 Bereits bestehende Kontakte nach Deutschland
}

Einige Alumni aus unseren Fallstudienländern haben sich darüber hinaus für ein Studium oder einen Forschungsaufenthalt in Deutschland entschieden, weil sie bereits davor akademische oder berufliche Kontakte nach Deutschland hatten. So knüpfte zum Beispiel eine kolumbianische Alumna während eines deutsch-kolumbianischen Forschungsprojektes, das von der GIZ finanziert wurde, Kontakte zu deutschen Kolleginnen an verschiedenen deutschen Universitäten. In diesem Rahmen entstand auch ihr Kontakt zu einem Professor an der Universität Münster, bei dem sie ihr späteres Promotionsstudium anfing: 
"Before the KAAD, I got a scholarship for my Master's thesis, and I was also working at the university. They gave me also the permission to go to Germany to study, and then I came back to Colombia, and I was also working at the university. During that time, it was for my Master's thesis from 2007 until 2010, and then I went back to Colombia, and I was continuing working together with Germany. In the KAAD they told us about that organization, it was together with the GIZ, in this I was doing some research. I applied for some funding, and I could get money for equipment for the university, and we could do this research project. And this project was related to my Master work. So, in that case I was continuing, or applying what I was doing in Germany and also had some contact with Hanover University, because one researcher from Hanover was visiting us because of this project. She was working together with us. So, it was really interesting. And then I met my actual supervisor. He is also from the University of Münster. So, I will get my Ph.D. from University of Münster. But I actually work with Helmholtz in Leipzig. This is an environmental center, and for my Ph.D. also I was trying to combine the research in Germany and research what is still missing in Latin America, and especially in my country in Colombia, and in my research area. My research is about pesticides [Pestizide]. I'm an environmental engineer. In my Master I worked about contermination in soil, and now in my Ph.D. I'm working on pesticides in soil. So my field is soil research" (Interview mit einer kolumbianischen Alumna, Münster, 2017).

Ein anderer kolumbianischer Alumnus wollte unbedingt für ein Studium zurück nach Berlin, weil er 2012 bereits schon ein sechsmonatiges Praktikum bei der kolumbianischen Botschaft gemacht und bereits Kontakte zu Professor ${ }^{\star}$ innen in Berlin geknüpft hatte (Interview mit einem kolumbianischen Alumnus, Skype, 2017).

Bei den ehemaligen indonesischen Stipendiat*innen verhält es sich ganz ähnlich. Dabei spielt auch die Partneruniversität des KAAD in Indonesien, die katholische Atma-Jaya Universität in Jakarta, eine besondere Rolle. Häufig werden Studierende der Atma-Jaya Universität als Stipendiat*innen des KAAD über das Partnerauswahlgremium des KAAD in Indonesien ausgewählt. So wurde zum Beispiel einer Alumna, die bereits damals als Juradozentin an der Atma-Jaya Universität arbeitete, von der Vorsitzenden des KAAD-Partnergremiums, die zugleich auch Stellvertretende Dekanin der Atma-Jaya Universität ist, vorgeschlagen, für eine Jurapromotion nach Deutschland zu gehen. Die Wahl fiel letztlich auf ein Promotionsstudium an der Ludwig-Maximilians-Universität, weil dort einer der führenden Professoren im internationalen Wettbewerbsrecht lehrte. Dafür bekam sie auch ein KAAD-Stipendium (Interview mit einer indonesischen Alumna, Jakarta, Indonesien, 2017). 
Eine andere Dozentin im Fachbereich Psychologie an der Atma Jaya Universität wurde ebenfalls aufgrund der engen Bindungen der Universität mit dem KAAD für ein Promotionsstudium in Deutschland vorgeschlagen. Eine weitere Stipendiatin entschied sich für ein Studium an der Universität Erlangen-Nürnberg, weil sie zuvor Kontakte dorthin knüpfte, als sie an einem Forschungszentrum in Jakarta arbeitete, das ein Kooperationspartner der Universität Nürnberg-Erlangen ist (Interview mit einer indonesischen Stipendiatin, Skype, 2017). Ein Alumnus wurde von seinem Professor an der Universität Bandung an die TU Dresden vermittelt, weil er bereits mehrere Jahre mit Kolleg*innen dort zusammenarbeitete und die Forschung dort in seinem technischen Bereich weltweit führend ist (Interview mit einem indonesischen Alumnus in Deutschland, Skype, 2017).

Ein georgischer Alumnus nutzte seine langjährigen Forschungskontakte nach Deutschland, um zwei vom KAAD geförderte Forschungsaufenthalte an der Universität Tübingen und der Ludwig-Maximilians-Universität München durchzuführen. Diese Aufenthalte nutzte er, um Primärquellenforschung mit deutschen Kolleg*innen zur evangelischen Religionsgeschichte in Deutschland und Georgien zu betreiben. Bereits vor diesen Forschungsaufenthalten war er mit Unterstützung eines DAAD-Forschungsstipendiums in Deutschland und plant auch seine nächsten Forschungsaufenthalte mit DAAD- und KAAD-Stipendien zu finanzieren.

In Palästina wiederum gab es nur wenige KAAD-Alumni, die bereits vor ihrem Studien- oder Forschungsaufenthalt akademische oder berufliche Kontakte nach Deutschland hatten. Eine Ausnahme ist ein Sprachwissenschaftler an der Bethlehem Universität, der vom KAAD für einen zweimonatigen Forschungsaufenthalt an der Universität Göttingen gefördert wurde. Zu diesem Forschungsaufenthalt wurde er von einem Arabistikprofessor eingeladen, den er bereits vor über 20 Jahren während einer Tagung in Jerusalem kennenlernte.

Neben den beruflichen und universitären Kontakten haben sich Stipendiat*innen und Alumni auch aufgrund privater Verbindungen für ein Studium oder einen Forschungsaufenthalt in Deutschland entschieden. Dies gilt vor allem für die ghanaischen und kolumbianischen Stipendiat*innen und Alumni. So hat sich zum Beispiel ein ghanaischer Stipendiat für ein Studium in Deutschland entschieden, weil bereits seine Brüder in Leipzig studiert haben und dort sehr zufrieden waren (Interview mit einem ghanaischen Stipendiaten, Skype, 2017). Eine andere Stipendiatin aus Ghana ist nach Deutschland gegangen, weil ihr Eheman bereits im Jahr 2014 ein Promotionsstudium an der Universität Bayreuth begonnen hatte, wofür er ein Stipendium von der Universität Bayreuth bekommen hat, und wo sie dann ebenfalls ein Masterstudium aufnehmen konnte (Interview mit einer ghanaischen Stipendiatin, Bonn, 2017). Ein anderer Alumnus besuchte im Jahr 2003 die katholische Partnergemeinde der ghanaischen Stadt Tamale in Münster. Dort 
knüpfte er Freundschaften und Kontakte zu Mitgliedern der Partnergemeinde. Zudem entwickelte er aufgrund des Besuchs ein Interesse an Deutschland und der deutschen Kultur. Insbesondere von der sprichwörtlichen Pünktlichkeit der Deutschen und dem hohen Entwicklungsstand der Technik in Deutschland war er fasziniert. Deswegen ist er später nach Deutschland gegangen, um im Fach Medizin zu promovieren (Interview mit einem ghanaischen Alumnus, Tamale, Ghana, 2018). Ein ghanaischer Alumnus aus der Stadt Kumasi wollte unbedingt in Deutschland studieren, weil er bereits über seine Familie enge Beziehungen nach Deutschland hatte. In seiner Kindheit hat er viel über die deutsche Geschichte und Kultur von seinem Großvater gelernt. Sein Großvater, der das deutsche Bundesverdienstkreuz verliehen bekam, hatte Deutsch bei einem deutschen Missionar in Ghana gelernt und dann selbst Deutsch in einer deutschen Schule in Accra unterrichtet. Zudem empfahl ihm ein Cousin, der an der KNUST in Kumasi Biochemie lehrte und zuvor selbst in Deutschland studierte, ein Studium in Deutschland aufzunehmen (Interview mit einem ghanaischen Alumnus, Accra, Ghana, 2018).

Eine kolumbianische Alumna ist im Jahr 2006 mit ihrem Mann, der ein Promotionsstudium an der Universität Göttingen aufnahm, nach Deutschland gezogen. Ein halbes Jahr später nahm sie auch ein Promotionsstudium im Fach Psychologie auf. Dafür bewarb sie sich von Deutschland aus für ein Stipendium beim KAAD (Interview mit einer kolumbianischen Alumna, Medellin, Kolumbien, 2017). Eine andere kolumbianische Alumna folgte ebenfalls ihrem Freund nach Deutschland, der an der Universität Regensburg ein Jurastudium absolvierte und anschließend promovierte. Später promovierte sie ebenfalls im Fach Rechtswissenschaften an der Universität Regensburg (Interview mit einer kolumbianischen Alumna, Bogotá, Kolumbien, 2017). Eine andere kolumbianische Alumna entschied sich für ein Studium in Heidelberg, weil sie bereits direkt nach dem Abitur im Jahr 2003 als Austauschschülerin nach Heidelberg kam und noch Freundschaften nach Heidelberg pflegte. Zudem hat ihr die Stadt sehr gut gefallen, die auch eine hohe Lebensqualität für Studierende biete. Das Schüler*innenaustauschprogramm zwischen ihrer Schule in Medellin und einer Schule in Heidelberg war im Jahr 2002 von deutschen Lehrer*innen gegründet worden, um Straßenkindern in Kolumbien mehr Bildungschancen und internationale Erfahrungen zu ermöglichen (Interview mit einer kolumbianischen Alumna, Heidelberg, 2018).

Eine indonesische Alumna entschied sich für ein Studium in Deutschland, weil bereits ihr Onkel und ihre Cousine in Deutschland lebten und ihr bei der Wohnungssuche und beim Einleben in Deutschland helfen konnten (Interview mit einer indonesischen Alumna, Jakarta, Indonesien, 2017). Eine andere indonesische Alumna zog mit ihrem Ehemann nach Deutschland, mit dem sie bereits in Indonesien an derselben Universität studiert hatte und der auch ein KAAD-Stipendium 
für sein Studium in Deutschland bekam. Sie bewarb sich auch für ein Studium in den Niederlanden, entschied sich aber letztlich für Deutschland (Interview mit einer indonesischen Alumna, Jakarta, Indonesien, 2017).

Ähnlich war es auch bei den palästinensischen und georgischen Alumni. So entschied sich z. B. eine palästinensische Alumna bewusst für ein Studium in Berlin, weil bereits ihre Schwester dort studierte und immer noch dort lebt. Ein aktueller georgischer KAAD-Stipendiat ging ebenfalls nach Berlin zum Studieren, weil ein Freund, der ebenfalls vor ein paar Jahren dort studierte, ihn von den Vorzügen der Freien Universität und der Weltstadt Berlin überzeugte. Zudem planen auch weitere seiner Freunde in Georgien in Zukunft ein Studium in Berlin aufzunehmen.

\subsubsection{Persönliches Interesse an Deutschland}

Ein persönliches Interesse an Deutschland spielte für die Entscheidung, ein Studium in Deutschland aufzunehmen, ebenfalls eine Rolle. Häufig hatte dieses Interesse auch etwas mit der Studienwahl im Herkunftsland zu tun. Ein kolumbianischer Alumnus studierte z. B. bereits deutsche Literatur in Kolumbien und entschied sich deshalb auch bewusst für ein Studium in Deutschland:

„Das war vor allem meine Begeisterung für deutsche Literatur. Ich konnte zwar schon etwas Deutsch, aber es war noch nicht so gut, um es in Deutschland studieren zu können. In Deutschland habe ich gutes Deutsch gelernt und kann es heute in Seminaren an der Uni hier in Bogotá unterrichten. Deswegen bin in ich damals nach Deutschland gegangen" (Interview mit einem kolumbianischen Alumnus, Bogotá, Kolumbien, 2017).

Ein anderer Kolumbianer hatte sich für ein Studium in Deutschland aufgrund seines Interesses für das deutsche Bankenwesen, das bereits ein Teil seiner Spezialisierung in seinem Jurastudium in Kolumbien war, entschieden:

"Ich bin nach Deutschland gegangen, weil das deutsche Bankenrecht damals das Vorbild für das kolumbianische Bankenrecht geworden ist. Im Hinblick auf diese Vorbildfunktion bin ich dahin geflogen mit dem Ziel ein Studium in diesem Bereich sehr stark zu vertiefen und um dann damit in Kolumbien weiter arbeiten zu können. [...] Ich bin in Deutschland angekommen, um dieses Studium durchzuführen, allerdings hatte ich vorher überhaupt keinen Kontakt zu Deutschland, hatte ich doch vorher immer ein großes Interesse an der deutschen Kultur. Dieses Interesse ist vielleicht darauf zurückzuführen, dass mein Name 
Helmut ist. Das war sehr seltsam, weil meine Eltern überhaupt keine Verbindungen zu Deutschland hatten. Allerdings haben sie diesen schönen Namen für mich ausgewählt. Allerdings habe ich während meines Studiums immer daran gedacht, einmal nach Frankreich zu fliegen und dort ein Aufbaustudium auszuführen, weil das kolumbianische BGB aus dem französischen Code Civil stammt. Erst nachdem ich bei der kolumbianischen Bankenaufsichtsbehörde zu arbeiten angefangen habe und dort festgestellt habe, dass das deutsche Bankenrecht sehr wichtig für das kolumbianische Bankenrecht war, habe ich das erste Mal daran gedacht, Frankreich ist nicht richtig, richtig ist es nach Deutschland zu gehen. Demgemäß habe ich angefangen Deutsch zu lernen"(Interview mit einem kolumbianischen Alumnus, Bogotá, Kolumbien, 2017).

Eine indonesische Stipendiatin hat sich für ein Musikstudium in Deutschland entschieden, weil sie sich schon seit ihrer Kindheit für klassische deutsche Musik interessiert hat. Ihre drei Lieblingskomponisten kommen alle aus Deutschland: Bach, Beethoven und Händel. Zudem gefällt ihr der deutsche Ansatz der Musikvermittlung an den deutschen Hochschulen, der ihrer Meinung nach direkter, klarer und zielorientierter ist als der US-amerikanische oder britische Ansatz. Um klassische deutsche Musik zu studieren, musste sie ins Ausland gehen, weil das in Indonesien nicht möglich ist (Interview mit einer indonesischen Stipendiatin, Skype, 2017).

Auch einen ghanaischen Stipendiaten zog das große Interesse für klassische Musik nach Deutschland. Er wollte vor allem nach Deutschland, um die Musik seines großen Vorbilds, Johann Sebastian Bach, auf den Originalorgeln in deutschen Kirchen zu hören, auf denen schon Bach spielte. Er selbst ist sehr engagiert und spielt regelmäßig Orgel zu den Sonntagsgottesdiensten in seiner Heimatgemeinde in Ghana (Interview mit einem ghanaischen Stipendiaten, Skype, 2017).

Das persönliche Interesse für ein Studium in Deutschland entstand auch daher, dass einige Alumni bereits eine deutsche Schule im Herkunftsland vor ihrem Studium in Deutschland besuchten. Unsere Online-Umfrage ergab, dass jeder siebte KAAD-Alumni bereits eine deutsche Schule im Herkunftsland besucht hatte (50 der 354 Befragten).

\subsubsection{Weitere Gründe}

Manchmal spielten auch mehrere Gründe gleichzeitig eine Rolle, warum sich eine Alumna bzw. ein Alumnus für ein Studium in Deutschland entschieden hat. Eine kolumbianische Alumna hat sich sowohl aus familiären als auch aus wissenschaftlichem Interesse für Deutschland entschieden: 
„Es gibt verschiedene Gründe. Das erste war, dass meine Schwester seit 25 Jahren in Deutschland wohnt. Ein Teil meiner Familie wohnt in Deutschland, meine Schwester und zwei Nichten. Ich hatte viel Kontakt mit Deutschland. Und ich finde, Deutschland ist ein sehr gutes Land zu studieren. Ich war 29 Jahre alt, ich war an der Heidelberg Universität und Frankfurt Universität, ich konnte perfekt studieren. Viele Leute haben mir vorher erzählt über die besonderen Stipendien, Kultur, die Infrastruktur und die guten Unis in Deutschland. Aber ich fand die Sprache sehr kompliziert. [...] Ich habe Soziologie an der Universidad de Colombia studiert und Politische Philosophie an der Universidad Javeriana, und die Menschen von der Literatur und Soziologie und Philosophie ist in deutscher Sprache. Die Tradition von Soziologie und Philosophie ist in deutscher Sprache. Deswegen fand ich es sehr interessant diese beiden Fächer in Deutschland zu studieren"(Interview mit einer kolumbianischen Alumna in Bogotá, Kolumbien, 2017).

Eine andere Kolumbianerin hat sich für ein Studium in Deutschland entschieden, weil das Studium eine gute Qualität hat und gleichzeitig relativ wenig kostet. Zudem interessierte sie sich für deutsche Geschichte und Kultur:
"I was already thinking to do my Master abroad since 2012. I was thinking about Spain - of course, because of the language - but I needed a scholarship, because I did not have enough money to study abroad. At the end, I decided to go to Germany, because the university system is good, you don't have to pay much for the education, and it is still a good quality. Also, the reputation of Germany is very good worldwide, also in Colombia we know about the good universities in Germany. I'm also very interested in German history and culture. These are the reasons why I wanted to go to Germany." (Interview mit einer kolumbianischen Alumna in Bogotá, Kolumbien, 2017).

Diese Beispiele zeigen, dass es vielfältige Gründe gibt für die Entscheidung ein Studium oder ein Forschungsaufenthalt in Deutschland zu absolvieren. Dabei spielen neben Karriereplanung und beruflichen Zielen eben sehr häufig auch ganz private Gründe, wie Familienbeziehungen oder Freundschaften in Deutschland eine Rolle. Dennoch war interessant zu erfahren, dass für einige der befragten Stipendiat*innen, Deutschland sozusagen nur „zweite Wahl“ war. Viele wären am liebsten in die USA, Großbritannien oder nach Kanada gegangen, um dort ein Studium aufzunehmen, vor allem weil es sich dabei um englischsprachige Länder handelt, die eine gute internationale Reputation genießen. Letztendlich haben sie sich aber für Deutschland entschieden, weil das Studium hier kostengünstiger ist. In 
anderen Ländern, wie den USA oder Großbritannien, seien die Aufnahmehürden, insbesondere die Studiengebühren zu hoch.

\subsection{Bewerbung beim KAAD}

Im Gegensatz zum DAAD ist der KAAD kein akademisches Stipendienprogramm, das aktiv Werbung im In- und Ausland für seine Stipendien betreibt. Eher im Gegenteil: Der KAAD versteht sich mehr als eine „kleine akademische Familie“, die potenzielle Stipendiat*innen über Mundpropaganda durch Menschen mit einem Bezug zum KAAD findet. Nach den Ergebnissen unserer quantitativen OnlineBefragung erfuhren auch die Alumni mehrheitlich nicht durch die Institutionen oder Partnerorganisationen des KAAD von der Möglichkeit ein KAAD-Stipendium zu bekommen, sondern eher durch Freund*innen und Bekannte, die den KAAD kennen, entweder weil sie selbst einmal KAAD-Stipendiat*innen waren oder welche kennen, die einmal Stipendiat*innen gewesen waren. So haben über die Hälfte (56\% oder 266 von 480 Befragten) aller befragten Alumni und aktuellen Stipendiat ${ }^{*}$ innen angegeben, dass sie durch Freund ${ }^{*}$ innen oder Bekannte, die selbst KAAD-Stipendiat*innen oder durch Freunde/Bekannte, die den KAAD kennen, aber keine Stipendiat*innen waren oder sind, vom KAAD erfahren haben. Die Interviews mit 135 Alumni und aktuellen Stipendiat*innen haben zu ähnlichen Ergebnissen geführt. Die meisten Alumni haben von Verwandten oder Bekannten vom Stipendienprogramm des KAAD erfahren. Eine kolumbianische Alumna erfuhr zum Beispiel vom KAAD von ihrem Ehemann, der sich ebenfalls um ein Stipendium beworben hatte:

"Ich bin auf den KAAD gekommen, weil ich sehr engagiert in der Kirche bin, auch schon in Kolumbien damals. Mein Katholischsein ist mir sehr wichtig. Ich habe auch meinen Mann in der Kirche kennengelernt. Von ihm habe ich auch vom KAAD erfahren, er hatte sich auch schon um ein Stipendium dort beworben, es damals aber nicht bekommen. Dafür bekam er später dann ein Stipendium vom DAAD. Er hatte damals online für Stipendienmöglichkeiten recherchiert und dabei auch den KAAD gefunden. Er hat im Fach Veterinärmedizin studiert und promoviert" (Interview mit einer kolumbianischen Alumna in Medellin, Kolumbien, 2017). 
Eine indonesische Stipendiatin erfuhr von einer guten Freundin von der KAADFörderung:

"A friend of mine got a scholarship from the KAAD, and she recommented me to get one too, because she was very happy with her scholarship" (Interview mit einer indonesischen Stipendiatin, Skype, 2017).

Andere Verwandte oder Bekannte, von denen die Alumni von der Möglichkeit erfahren haben, sich um ein Stipendium beim KAAD zu bewerben, waren zum Beispiel ein Cousin (Kolumbianerin), ein Kommilitone (Indonesier), ein Priester (Georgierin) oder ein Vertrauensprofessor an der Universität im Herkunftsland (Ghanaer).

Am zweithäufigsten haben die interviewten Alumni von Mitgliedern des KAAD-Partnergremiums im Herkunftsland gehört. Dies geht auf die engen Kontakte zwischen den Länderreferaten des KAAD in Bonn und den Partnergremien in den Herkunftsländern, die eine Vorauswahl unter den Bewerber*innen für ihr Land treffen, zurück. Dabei sind die Partnergremien oftmals an der katholischen Partneruniversität im Herkunftsland ansässig. So bekam ein ghanaischer Alumnus vom KAAD-Partnergremium an der Partneruniversität KNUST in Kumasi, Ghana, während seines Bachelorstudiums den Hinweis, sich beim KAAD um ein Stipendium zu bewerben (Interview mit einem ghanaischen Alumnus in Kumasi, Ghana, 2018). Das Stipendium des KAAD hat er sogar einer Finanzierung des renommierten staatlichen Stipendienwerk GETFund (Ghana Education Trust Fund) vorgezogen. Aufgrund der ghanaischen Kolonialgeschichte, zahlreicher traditioneller Verbindungen und der hohen Zahl ghanaischer Studierender in England, vergibt GETFund aber vor allem Stipendien für ein Studium in Großbritannien. Zudem bewerben sich viele Ghanaerinnen beim KAAD um ein Stipendium für einen Master- oder Promotionsstudiengang in Deutschland, die bereits schon im Rahmen des Sur-Place-Stipendienprogramms ${ }^{10}$ für Bachelorstudierende an ghanaischen Universitäten vom KAAD gefördert wurden.

Ferner erfuhren die KAAD-Alumni, die sich von Deutschland aus um ein Stipendium beworben haben, vom KAAD durch die katholische Hochschulgemeinde (KHG). Die folgenden Aussagen dreier kolumbianischer Alumni machen deutlich, dass die Mitarbeiter der KHG Beratungen und Informationsveranstaltungen zum Stipendienprogramm des KAAD anbieten:

10 Das Sur-Place-Stipendienprogramm des KAAD fördert Studierende im Rahmen ihres Bachelorstudiums im Herkunftsland, vor allem in afrikanischen Ländern. 
„Mitarbeiter in der KHG haben mir das empfohlen. Es gab damals auch einen sehr netten Mitarbeiter, ich glaube ein Vertrauensmann des KAAD, der mich über die Stipendienmöglichkeiten des KAAD beraten hat. Er hat auch das erste Bewerbungsgespräch mit mir gemacht"(Interview mit einer kolumbianischen Alumna in Bogotá, Kolumbien, 2017).

„Es gibt eine KHG in Gießen und ich bin immer dorthin gegangen für die Gottesdienste und zu manchen Leuten da. Einer der Leute dort hat über Stipendium des KAAD einen Vortrag gehalten, und ich habe gedacht, ich könnte dieses Stipendium bekommen, weil ich hatte nicht so viel Geld, und ich habe mich beworben. Ich wurde dann ein Jahr und drei Monate vom KAAD gefördert" (Interview mit einer kolumbianischen Alumna in Deutschland, Skype, 2017).

"I have a friend in the program, who is also a KAAD scholar. She is from Simbabwe. We both are Catholic, and she invited me to the KHG. And there I asked for an appointment, and they told me all about the scholarship. And I did my application for the KAAD throught the contact person at the KHG" (Interview mit einer kolumbianischen Alumna in Deutschland, Skype, 2017).

Eine andere Alumna aus Kolumbien erfuhr vom KAAD während eines Deutschkurses, den sie am Goethe-Institut in Bogotá belegte:

"I found out about the KAAD in the German class at the Goethe Institute in Bogotá. A friend in my class at the Goethe Institute told me about the KAAD. After the class, I contacted Krüggeler by e-mail that I found on the webpage of the KAAD. And Krüggeler told me to write Carlsos Henao who is the head of the partner committee of the KAAD in Colombia. He wrote back directly and sent me all the requirements for the application. They answered very fast and had good advices. They helped me a lot" (Interview mit einer kolumbianischen Alumna in Bogotá, Kolumbien, 2017).

Der Bewerbungsprozess selbst um ein KAAD-Stipendium unterscheidet sich deutlich zwischen den zwei Stipendienprogrammen des KAAD, also ob man sich vom Herkunftsland aus (Stipendienprogramm 1) oder in Deutschland (Stipendienprogramm 2) bewirbt.

Im Herkunftsland findet die Bewerbung über das Partnergremium des KAAD statt, das eine Vorauswahl der Bewerber*innen trifft und Empfehlungen von Bewerber*innen für ein Stipendium an den KAAD und den Auswahlausschuss in Deutschland abgibt. Um die konkreten Schritte der Bewerbung deutlich zu machen, 
erläutern wir im Folgenden den Prozess am Beispiel einer ghanaischen Bewerberin, die sich von Ghana aus für ein Stipendium beworben hat (S1-Programm). Zuerst musste sie alle notwendigen Bewerbungsunterlagen sammeln (Motivationsschreiben, CV, Empfehlungsschreibens einer Professorin*eines Professors der Universität Tamale - ein Schreiben eines deutschen Professors ${ }^{\star}$ einer deutschen Professorin ist nicht notwendig - beglaubigte Zeugnisse, Empfehlungsschreiben eines katholischen Pfarrers, eine Notenübersicht des BA-Studiums und die BA-Abschlussarbeit). Für die Bewerberin waren das sehr viele Unterlagen und sie hat mehrere Monate gebraucht, um alle Unterlagen zu besorgen. Nach Einreichen der Unterlagen hat es weitere zwei Monate gedauert, bis sie zu einem ersten Bewerbungsgespräch des KAADAuswahlgremiums eingeladen wurde. Dafür wurde sie nach Kumasi eingeladen. Die Kosten für die rund zehnstündige Busfahrt von Tamale nach Kumasi und die Übernachtungskosten in Kumasi wurden vom Partnergremium übernommen (wurde also mit Geldern des KAAD finanziert). Das Auswahlgespräch mit vier Mitgliedern des Partnergremiums (bestehend aus drei KAAD-Alumni, die jetzt Professoren an der Kumasi-Uni sind und zwei katholischen Pfarrern; ein Pfarrer konnte nicht kommen) dauerte ca. eine Stunde und verlief sehr gut. Es wurden hauptsächlich Fragen zu ihrer Motivation nach Deutschland zu gehen, ihrem Glauben und religiösem Engagement sowie ihrem Studium in Tamale und Vorstellungen über das Studium in Deutschland gestellt. Am gleichen Tag wurden noch fünf weitere Bewerber*innen um ein KAAD-Studium für Bewerbungsgespräche vom Partnergremium nach Kumasi eingeladen. Nach vier Wochen bekam sie die Nachricht vom Partnergremium, dass sie für die nächste Bewerbungsrunde ausgewählt wurde und ihre Unterlagen nach Deutschland geschickt werden. Dort haben dann die Mitarbeitenden des KAAD eine weitere Auswahl getroffen und ihre Auswahl für ein Stipendium an den akademischen Ausschuss des KAAD (bestehend aus deutschen Professor*innen und Vertrauensdozent*innen) weitergegeben, die die endgültige Entscheidung für die Vergabe von Stipendien treffen. Die endgültige Zusage für ihr Stipendium hat die Bewerberin nach ca. fünf Monaten erhalten (Interview mit einer ghanaischen Stipendiatin, Bonn, 2017).

Für die Bewerber*innen, die sich von Deutschland aus bewerben, treffen die Katholischen Hochschulgemeinden an den Universitäten vor Ort die Vorauswahl und geben ihre Empfehlungen an den KAAD und den Auswahlausschuss weiter. Auch hier kann der Bewerbungsprozess bis zu vier und sechs Monaten dauern. In Ausnahmefällen aber auch bis zu einem Jahr. Dies wurde von einigen Bewerber*innen auch bemängelt, da der Bewerbungsprozess um Studienplatz und Stipendium in Deutschland für viele sehr schwierig war, alle notwendigen Bewerbungsunterlagen im Herkunftsland beizuschaffen: 
"The application process was very long and complicated. It took me one year to collect all the application documents. For the application, I needed the invitation letter from a German university, and a letter from a priest in Bogotá. That was very expensive and took a lot of time, because I had also to drive to all these people, they wanted to see me and talk to me. They did not just send me the forms and letters. They had a lot of questions, but I understand, they wanted to know me. [...]

And also a big problem: it took a lot of time to get the letter from the German university, from Essen. I needed two letters of recommendation, one of the Professor at the university in Bogotá and from the United Nations that was easy to get. However, the whole application process took a long time, and sometimes I was frustrated, of course" (Interview mit einer kolumbianischen Alumna in Bogotá, Kolumbien, 2017).

Bei dem aufwendigen Bewerbungsprozess wurde auch bemängelt, dass den deutschen Behörden alle notwendigen Unterlagen ausschließlich auf Deutsch vorgelegt werden dürfen. Dies erschwerte vielen Alumni die Bewerbung, weil bei den meisten zu diesem Zeitpunkt die Deutschkenntnisse noch nicht so gut sind. Eine indonesische Stipendiatin merkte dazu an:

"The whole process of application and the communication with German bureaucrats was very long, and it was sometimes frustrating, because I did not understand the paper stuff, I did not understand German. Everything was very complicated. Also, later in Dresden it was difficult to communicate with the people in the city offices, because they don't speak English and seem to be unfriendly and impatient with people who don't understand their culture and language. But I have heard that even Germans, I mean German citizens have problems with their bureaucrats, and the special German Juristensprache" (Interview mit einer indonesischen Stipendiatin, Skype, 2017).

Es gab aber auch positive Stimmen zum Bewerbungsprozess:

"Die Bewerbung ging ziemlich schnell und fand ich auch einfach. Auch ziemlich unkompliziert. Im gesamten Prozess wurde ich immer gut informiert und wusste was ich zu machen hatte" (Interview mit einer indonesischen Stipendiatin, Skype, 2017). 
„Die Bewerbung war sehr unkompliziert und hatte circa sechs Monate gedauert. Die Vertrauensperson des KAAD bei der KHG hatte mich sehr gut im Bewerbungsprozess beraten" (Interview mit einer ghanaischen Stipendiatin, Skype, 2017).

Bei der Auswahl der Stipendiat*innen achtet der KAAD auch darauf, ob man die Bewerber*innen schon kennt und bereits ein Vertrauensverhältnis besteht. Demnach gab es bereits schon mehrere Fälle, in denen Alumni mehr als einmal vom KAAD gefördert wurden, so zum Beispiel einmal im Rahmen des Sur-place-Stipendienprogramms im Heimatland und dann für einen Master- oder Promotionsstudiengang in Deutschland (wie z.B. bei mehreren ghanaischen Alumni) oder für ein Master- und ein Promotionsstudium in Deutschland oder für zwei oder mehrere Forschungsaufenthalte im Rahmen des Postdocs oder der Professur in Deutschland, wie dies zum Beispiel bei mehreren georgischen Forschenden der Fall war. Dahinter steckt die Idee, sich als eine Art „akademische Familie“ zu verstehen. Ein ghanaischer Alumnus, der sowohl für sein Master- als auch Promotionsstudium in Deutschland gefördert wurde, drückte dies auch entsprechend aus:

"Ich gehörte sozusagen schon zur Familie und es war schon ein gewisses Vertrauen da. Der KAAD wusste, dass ich mein Studium gut abgeschlossen hatte und mich gut in Deutschland verhalten hatte. Sie wussten auch, dass ich einen guten Job als Lecturer an der KNUST in Kumasi mache"(Interview mit einem ghanaischen Alumnus, Kumasi, Ghana, 2018).

In einigen Fällen kooperiert der KAAD auch mit den Partneruniversitäten in Bezug auf die Stipendienfinanzierung. So zahlt der KAAD in einigen Fällen nur 60 Prozent des Stipendiums, während die Partneruniversität für den Rest des Geldes aufkommt. Ein Beispiel für ein solches Arrangement ist die bereits erwähnte Atma Jaya Universität in Jakarta, Indonesien. Im Rahmen des Stipendienabkommens verpflichten sich die ausgewählten Stipendiat*innen dazu, nach dem geförderten Studium in Deutschland als Lehrkräfte oder Wissenschaftler*innen an die Partneruniversität zurückzukehren. Das Auslandsstudium bzw. der Gastaufenthalt in Deutschland dient damit sozusagen der Weiterbildung des eigenen Lehr- und Wissenschaftspersonals. Damit konnte zum Beispiel eine Juradozentin mit einem KAAD-Stipendium für vier Jahre zur Promotion an die Ludwig-Maximilians-Universität nach Deutschland. Nach ihrer Rückkehr verpflichtete sie sich allerdings für mindestens weitere 12 Jahre an der Atma Jaya Universität Jura zu lehren (Interview mit einer indonesischen Alumna in Jakarta, Indonesien, 2017). Ähnliche Abkommen bestehen auch mit den KAAD-Partneruniversitäten KNUST in Kumasi, Ghana, 
Bethlehem University in Palästina, Sulkhan-Saba-Universität Tiflis in Georgien sowie der Universidad Javeriana Bogotá und früher der Universidad Bolivariana in Kolumbien.

\subsection{Sprachkurs im Herkunftsland}

Um sich auf das Studium in Deutschland vorzubereiten besuchten einige Stipendiat ${ }^{\star}$ innen Deutschkurse im Heimatland, entweder an einer Universität oder einem Sprachinstitut wie dem Goethe-Institut. Dies gilt vor allem für diejenigen Alumni, die zunächst ein Studium ohne KAAD-Stipendium in Deutschland begonnen und sich erst später für ein Stipendium beworben haben, aber eben zunächst einen Nachweis für Grundkenntnisse in der deutschen Sprache benötigten, um ein Studium an einer deutschen Hochschule aufzunehmen.

Zwei ghanaische Alumni absolvierten jeweils einen Deutschkurs an einer Universität in Ghana: Für sechs Monate an der Cape Coast University und für zwei Jahre an der Legon University in Accra. Zwei weitere Ghanaer belegten jeweils einen Deutschkurs für sechs Monate mit sechs Stunden pro Woche am Goethe-Institut in Accra. Beide fanden den Deutschkurs sehr schwierig und zeitintensiv. Auch ein kolumbianischer Alumnus belegte einen Deutschkurs am Goethe-Institut in Bogotá bevor er zum Studium nach Deutschland ging:

„Ich habe 14 oder 16 Monate hier am Goethe-Institut Deutsch gelernt, wobei ich bedauerlicherweise keine Zeit hatte nach dem Unterrichtsbesuch Hausaufgaben und alles sofort zu erledigen, weil ich sehr beschäftigt war als Anwalt, als Jurist zu arbeiten" (Interview mit einem kolumbianischen Alumnus in Bogotá, Kolumbien, 2017).

Eine andere Kolumbianerin belegte sogar für vier Jahre einen Deutschkurs am Goethe-Institut in Bogotá:

„Vor meiner Reise habe ich etwa vier Jahre Deutsch am Goethe-Institut gelernt und dort hatte ich auch die Möglichkeit, kulturelle Aspekte zu lernen mit deutschen Professoren. Wir hatten Landeskunde und so. Und ich war ein bisschen vorbereitet für den Kulturschock. Das war nicht so stark für mich, ehrlich gesagt. Die größte Herausforderung für mich war die Sprache. Mir war bewusst, dass die Sprache ein Mittel war, um mich zu integrieren, war die Beherrschung der Sprache" (Interview mit einer kolumbianischen Alumna in Bogotá, Kolumbien, 2017). 
Die indonesischen Alumni belegten einen Deutschkurs im Herkunftsland entweder am Goethe-Institut oder am Studienkolleg in Jakarta jeweils für sechs Monate oder einem Jahr, um ein A1- und A2-Sprachniveau zu erreichen.

Einige palästinensische Alumni hingegen lernten bereits Deutsch in der Schule. So war z. B. ein Alumnus Schüler an der renommierten deutschen Schule „Talitha Kumi “ in Bait Dschala nahe Bethlehem, die 1950 vom Diakoniewerk Kaiserwerth gegründet worden war und an der überwiegend deutsche Lehrer*innen unterrichten (Talitha Kumi 2018). Dort lernte sie seit der ersten Klasse Deutsch und legte nach der zwölften Klasse die Deutsche Internationale Abiturprüfung (DIAP) ab. Trotz ihrer bereits sehr guten Deutschkenntnisse erwarb sie ein zusätzliches Sprachzertifikat beim DAAD, bevor sie ihr Studium an der Universität Heidelberg aufnahm. Mit der Qualität ihres Spracherwerbs in Palästina war sie sehr zufrieden:

"Die deutsche Schule in Bait Dschala ist sehr bekannt und man bekommt dort sehr gute Sprachkenntnisse vermittelt. Das ist die Nummer eins für Deutsch in Palästina. Auch sonst ist die schulische Ausbildung dort sehr gut. Meine Eltern haben mich dorthin geschickt, nicht nur um Deutsch zu lernen, sondern insgesamt eine gute Bildung zu bekommen. Dort ist auch meine Idee entstanden, in Deutschland zu studieren. Aber bevor ich dann endlich für ein Studium in Deutschland zugelassen wurde, wollte ich mir meine guten Deutschkenntnisse noch einmal vom DAAD in Palästina bestätigen lassen, damit ich auch gute Chancen für ein Stipendium habe" (Interview mit einer palästinensischen Aluma in Deutschland, Skype, 2016).

Open Access Dieses Kapitel wird unter der Creative Commons Namensnennung 4.0 International Lizenz (http://creativecommons.org/licenses/by/4.0/deed.de) veröffentlicht, welche die Nutzung, Vervielfältigung, Bearbeitung, Verbreitung und Wiedergabe in jeglichem Medium und Format erlaubt, sofern Sie den/die ursprünglichen Autor(en) und die Quelle ordnungsgemäß nennen, einen Link zur Creative Commons Lizenz beifügen und angeben, ob Änderungen vorgenommen wurden.

Die in diesem Kapitel enthaltenen Bilder und sonstiges Drittmaterial unterliegen ebenfalls der genannten Creative Commons Lizenz, sofern sich aus der Abbildungslegende nichts anderes ergibt. Sofern das betreffende Material nicht unter der genannten Creative Commons Lizenz steht und die betreffende Handlung nicht nach gesetzlichen Vorschriften erlaubt ist, ist für die oben aufgeführten Weiterverwendungen des Materials die Einwilligung des jeweiligen Rechteinhabers einzuholen. 